\title{
La enseñanza de los instrumentos de cuerda frotada en la práctica de aula: 15 años de tesis doctorales españolas
}

\section{The Teaching of Bowed String Instruments in Classroom Practice: 15 years of Spanish Doctoral Dissertations}

\author{
Roberto Macián-González \\ rmacian@uji.es \\ Departamento de Educación y Didácticas Específicas \\ Universitat Jaume I \\ Castellón de la Plana, España \\ ORCID: http://orcid.org/0000-0002-5765-617X
}

doi: 10.7203/LEEME.43.13686

Recibido: 29-12-2018 Aceptado: 07-05-2019. Contacto y correspondencia: Roberto Macián González, Departamento de Educación y Didácticas Específicas, Universidad Jaume I. Avda. Vicent Sos Baynat, s/n, 12071 Castellón de la Plana, España.

\section{Resumen}

Los estudios de doctorado permiten a muchos profesionales de la educación indagar en su práctica para mejorarla. Los docentes pueden acudir a las distintas bases de datos y orientarse sobre cómo enfocar sus investigaciones. Para evitar posibles problemas al trabajar con las bases de datos, se puede acudir a los estudios de revisión de tesis doctorales. En la enseñanza de los instrumentos de la familia del violín, no se ha llevado a cabo este tipo de estudios. Este trabajo realiza un análisis biliométrico y una revisión de contenido de las tesis doctorales realizadas en España en los últimos 15 años. Para ello, se acudió a la base de datos Teseo, buscando trabajos cuya finalidad última fuera la aplicación de sus hallazgos en la práctica diaria del aula de instrumento. Por un lado, el objetivo de este estudio fue aportar datos estadísticos de interés. Por otro, fue la revisión y presentación de recomendaciones de fácil acceso para los profesores de estas disciplinas instrumentales. Los resultados de este trabajo muestran que se trata de un área novedosa en España y que ha experimentado un auge en los últimos años. Asimismo, se encontró que existen dos enfoques en las tesis estudiadas: las propuestas metodológicas y las investigaciones empíricas en el aula. Aunque estas tesis suponen valiosas contribuciones aportando hallazgos relevantes, se pone de manifiesto la necesidad de incrementar este tipo de investigaciones, pues suponen un porcentaje muy escaso de tesis relacionadas con la enseñanza musical.

Palabras clave: Tesis doctorales españolas, enseñanza del violín, base de datos TESEO.

\begin{abstract}
Doctoral studies allow many professionals to look into ways of improving their practice. Students can check out various databases guide themselves on how to focus their research. In order to help them, teachers can check some databases and assist in coping with problems they encounter. In order to avoid troubles, they can also take into account review studies of doctoral dissertations. These kind of studies have never been done in relation to the violin-family instrumental teaching. For this reason, this work provides new methods for working in this field by performing a bibliometric analysis and a content review of Spanish doctoral dissertations in the last 15 years. For this purpose, Teseo database was consulted, looking for studies whose finality was to apply its findings to the common instrumental classroom practice. On one hand, the objective of this paper is to show interesting statistical data. On the other hand, it is to review and show easily accessible recommendations for teachers of these instruments. Results of this work demonstrate how new these ideas are in Spain and how they have reached a boom in the last years. Moreover, two approaches appear in theses studied: methodological proposals and empiric researches in the classroom. Although these dissertations provide valuable contributions and reveal outstanding findings, a low percentage of studies related to musical teaching continues to demonstrate the need of increasing this kind of research
\end{abstract}

Key words: Spanish doctoral dissertations, violin teaching, TESEO database.

@ROBERTO MACIÁN GONZÁLEZ. THE CONTENT OF THIS ARTICLE IS THE SOLE RESPONSIBILITY OF THE AUTHORS. THE REVISTA ELECTRÓNICA DE LEEME AND UNIVERSITAT DE VALĖNCIA ARE NOT LIABLE FOR ANY LEGAL ACTIONS THAT MAY ARISE INVOLVING THE ARTICLE'S CONTENT. REVISTA ELECTRÓNICA DE LEEME -LISTA ELECTRÓNICA EUROPEA DE MÚSICA EN LA EDUCACIÓN-HTTP://OJS.UV.ES/INDEX/PHP/LEEME/INDEX. ISSN: 1575-956. EDITORES: UNIVERSIDAD DE VALENCIA Y JESÚS TEJADA GIMÉNEZ. VISIBILIDAD DE ESTA REVISTA: SCOPUS, EMERGING SOURCES CITATION INDEX (CLARIVATE), EBSCO, CINDOC (CSIC), CITEFACTOR, COPAC, DIALNET, DICE (CSIC), DOAJ, E-REVISTAS (CSIC), EBSCO PREMIER, ERIH+, GALE CENGAGE LEARNING, IN-RECS, IRESIE, LATINDEX, MIAR, OCLC WORLDCAT, RESH, REDIB, RILM CORE JOURNALS, SUDOC, ULRICHS, ESTA REVISTA ESTA
PUBLICADA CON EL APOYO INSTITUCIONAL DE REDIRIS-CONSEJO SUPERIOR DE INVESTIGACIONES CIENTIFICAS Y ES DE ACCESO LIBRE. CREATIVE COMMONS LICENSE 4.O BY 


\section{Introducción}

La investigación es fundamental para el avance de cualquier disciplina, siendo los estudios de doctorado una forma de iniciarse en esta forma de generar conocimiento. De hecho, muchos profesores y maestros utilizan los estudios de tercer ciclo para la profundización y mejora de su propia práctica docente. Para estos profesionales, la investigación puede servir para la mejora de los distintos procesos de enseñanza-aprendizaje, utilizando mejores herramientas de intervención docente y ofreciendo una educación de mayor calidad (Pastor, 2002). Además de propiciar esta mejora, la investigación conlleva una especialización que puede repercutir en el futuro profesional de los enseñantes (Oriol, 2009), lo que puede guiar los procesos de investigación del profesorado hacia un tema determinado con un enfoque u otro. No obstante, decidir qué investigar y cómo hacerlo puede suponer un problema para los neófitos, no sabiendo hacia donde enfocar sus inquietudes profesionales. Las didácticas específicas en la enseñanza musical no son una excepción.

La búsqueda y estudio de investigaciones previas es una forma de facilitar esta toma de decisiones para los investigadores. Esto permite tener una idea clara de los trabajos realizados en el propio campo, permitiendo enfocar las investigaciones según las propias motivaciones (Oriol, 2009). Aunque las tesis suelen culminar con la publicación de los resultados en artículos y comunicaciones a congresos con los que informar a la comunidad científica, la publicación y el impacto de los artículos sobre educación musical es menor que en otras áreas análogas (Morales, Ortega, Conesa, Ruiz-Esteban, 2017). Por esta razón, acudir a las distintas bases de datos de tesis doctorales es una tarea fundamental. Uno de los registros españoles de tesis doctorales es Teseo, en el que se pueden consultar las tesis doctorales leídas desde 1976 (Ministerio de Educación, Cultura y Deporte, 2017).

Aunque es una herramienta muy útil, los profesores que acudan a Teseo pueden encontrarse con ciertos problemas a la hora de buscar tesis doctorales relacionadas con su disciplina. Un problema que puede surgir es que el nombre no esté bien escrito, lo que hace que la base de datos no devuelva todos los resultados que debería (Oriol, 2009, 2012). También es posible que la base de datos no devuelva algunos trabajos porque las universidades no hayan validado la ficha de dicha tesis, pues pueden tardar varios meses en hacerlo. Este lento proceso de actualización puede provocar que no aparezcan tesis doctorales del año en curso (Fernández, Torralbo y Vallejo, 2008) y se produzcan posibles sesgos en la información obtenida (Nagore, 2005). Otro problema es la gran cantidad de trabajos que contiene esta base de datos, lo que hace necesario fijar muy bien los criterios de búsqueda para realizarla de forma estratégica (Martínez, 2013), obteniendo resultados fiables y manejables.

Otra opción a la hora de buscar y acceder a las tesis doctorales de una determinada disciplina es acudir a estudios bibliométricos o revisiones de literatura. Aunque los dos tipos de estudios tienen objetivos diferentes (Escorcia, 2008; Manterola, Astudillo, Arias y Claros,

@ROBERTO MACIÁN GONZÁLEZ. THE CONTENT OF THIS ARTICLE IS THE SOLE RESPONSIBILITY OF THE AUTHORS. THE REVISTA ELECTRÓNICA DE LEEME AND UNIVERSITAT DE VALĖNCIA ARE NOT LIABLE FOR ANY LEGAL ACTIONS THAT MAY ARISE INVOLVING THE ARTICLE'S CONTENT. REVISTA ELECTRÓNICA DE LEEME -LISTA ELECTRÓNICA EUROPEA DE MÚSICA EN LA EDUCACIÓN.HTTP://OJS.UV.ES/INDEX/PHP/LEEME/INDEX. ISSN: 1575-956. EDITORES: UNIVERSIDAD DE VALENCIA Y JESÚS TEJADA GIMÉNEZ. VISIBILIDAD DE ESTA REVISTA: SCOPUS, EMERGING SOURCES CITATION INDEX (CLARIVATE), EBSCO, CINDOC (CSIC), CITEFACTOR, COPAC, DIALNET, DICE (CSIC), DOAJ, E-REVISTAS (CSIC), EBSCO
PREMIER, ERIH+, GALE CENGAGE LEARNING, IN-RECS, IRESIE, LATINDEX, MIAR, OCLC WORLDCAT, RESH, REDIB, RILM CORE JOURNALS, SUDOC, ULRICHS, ESTA REVISTA ESTÁ PUBLICADA CON EL APOYO INSTITUCIONAL DE REDIRIS-CONSEJO SUPERIOR DE INVESTIGACIONES CIENTIFICAS Y ES DE ACCESO LIBRE. CREATIVE COMMONS LICENSE 4.O BY 
2011), aportan datos estadísticos útiles y resultados de fácil acceso. Una muestra de la importancia de este tipo de estudios es la gran cantidad de trabajos realizados sobre la producción de tesis doctorales (Osca-Lluch, Haba, Fonseca, Civera y Tortosa, 2013). No obstante, puede darse el caso de que este tipo de investigaciones sobre producción científica no se haya llevado a cabo en un determinado campo, pues se ha producido un gran crecimiento de determinadas disciplinas y áreas específicas (Morales et al., 2017).

España es un ejemplo en este crecimiento, pues ha habido un aumento considerable en el número de tesis relacionadas con la música en las últimas décadas (Gillanders y Martínez, 2005), llegando a las 366 tesis doctorales entre 1998 y 2007 (Oriol, 2009) y a las 2.255 en diciembre de 2018 (Narejos, 2018). Asimismo, a finales del siglo pasado, se produjo una diversificación en las temáticas y un descenso del porcentaje de estudios musicológicos, predominantes hasta 1994 (Nagore, 2005). Sin embargo, pese a este cambio en las temáticas de investigación, la mayoría de estudios en el campo de la enseñanza musical versan sobre temas históricos, encontrándose una falta de estudios versados es la pedagogía instrumental (Gillanders y Martínez, 2005). De hecho, entre las 366 tesis citadas anteriormente, únicamente 5 estaban relacionadas con el "Aprendizaje y enseñanza de la música" desde una perspectiva instrumental (Oriol, 2009).

Para tratar de identificar líneas de investigación y poder establecer las posibles lagunas de información, ha habido un cierto número de estudios sobre la producción de tesis relacionadas con la música en España. Entre otras variables, estos estudios han estudiado la temática, el año de publicación, las universidades en las que se han llevado a cabo o los departamentos en los que se han realizado estas investigaciones. Resulta destacable la diversificación por temática: 26 según Oriol $(2009,2012)^{1}$ y 18 según Nagore (2005). En cuanto a las universidades más productivas, los resultados son prácticamente idénticos en los distintos trabajos realizados. En orden descendente de producción se encontrarían: Granada, Autónoma de Barcelona, Complutense de Madrid, Barcelona, Oviedo, Autónoma de Madrid, Valencia, Valladolid y Salamanca (Nagore, 2005), invirtiendo el orden entre Complutense de Madrid y Barcelona según Gillanders y Martínez (2005).

En otros países, ha habido un interés por abordar problemáticas más concretas, como el estudio de tesis relacionadas con la historia de la educación musical y la terapia musical (Preston y Humphreys, 2007) o con la enseñanza de los instrumentos de cuerda (Kantorski, 1995). Este último analizó un total de 252 trabajos defendidos entre 1936 y 1992 en Estados Unidos de América. Además de estudiar cuestiones estadísticas (fecha, tipo de grado o instrumentos), realizó una categorización por temáticas dentro de la enseñanza de los instrumentos de cuerda, siendo el violín y la "técnica/destreza" las temáticas más habituales. Sin

\footnotetext{
${ }^{1}$ En sus dos estudios, Oriol modifica dos de las categorías. Clasifica como "Informática” y "Musicoterapia” en 2009 y utiliza "Educación Especial" y "Nuevas tecnologías" en 2012. En ambos trabajos, el "Aprendizaje y enseñanza de la música" lo aborda como una categoría separada de las otras 25.
}

@ROBERTO MACIÁN GONZÁLEZ. THE CONTENT OF THIS ARTICLE IS THE SOLE RESPONSIBILITY OF THE AUTHORS. THE REVISTA ELECTRÓNICA DE LEEME AND UNIVERSITAT DE VALĖNCIA ARE NOT LIABLE FOR ANY LEGAL ACTIONS THAT MAY ARISE INVOLVING THE ARTICLE'S CONTENT. REVISTA ELECTRÓNICA DE LEEME -LISTA ELECTRÓNICA EUROPEA DE MÚSICA EN LA EDUCACIÓN-HTTP://OJS.UV.ES/INDEX/PHP/LEEME/INDEX. ISSN: 1575-956. EDITORES: UNIVERSIDAD DE VALENCIA Y JESÚS TEJADA GIMÉNEZ. VISIBILIDAD DE ESTA REVISTA: SCOPUS, EMERGING SOURCES CITATION INDEX (CLARIVATE), EBSCO, CINDOC (CSIC), CITEFACTOR, COPAC, DIALNET, DICE (CSIC), DOAJ, E-REVISTAS (CSIC), EBSCO
PREMIER, ERIH+, GALE CENGAGE LEARNING, IN-RECS, IRESIE, LATINDEX, MIAR, OCLC WORLDCAT, RESH, REDIB, RILM CORE JOURNALS, SUDOC, ULRICHS, ESTA REVISTA ESTÁ PREMIER, ERIH+, GALE CENGAGE LEARNING, IN-RECS, IRESIE, LATINDEX, MIAR, OCLC WORLDCAT, RESH, REDIB, RILM CORE JOURNALS, SUDOC, ULRICHS, ESTA REVISTA
PUBLICADA CON EL APOYO INSTITUCIONAL DE REDIRIS-CONSEJO SUPERIOR DE INVESTIGACIONES CIENTIFICAS Y ES DE ACCESO LIBRE. CREATIVE COMMONS LICENSE 4.O BY 
embargo, en España no se han llevado a cabo estudios sobre tesis doctorales de áreas musicales específicas como es la enseñanza de instrumentos de cuerda frotada. Este tipo de estudios concretos en cuanto a temática y geografía son de interés tanto para investigadores (para cubrir posibles lagunas) como para autoridades educativas (para establecer políticas de investigación) (Morales et al., 2017).

Por todo ello, se consideró pertinente realizar una revisión de las tesis doctorales relacionadas con los procesos de enseñanza-aprendizaje de los instrumentos de cuerda frotada de la familia del violín (ICFFV ${ }^{2}$ de aquí en adelante) en España. Esta revisión se abordó desde dos puntos de vista: 1) un análisis bibliométrico para conocer algunos datos estadísticos de interés en la investigación en este campo; y 2) una revisión de los resultados más relevantes de las tesis estudiadas. Por un lado, el objetivo fue ofrecer una herramienta a los futuros investigadores, aportando propuestas metodológicas sobre las que realizar sus estudios. Por otro, se pretendió ofrecer una síntesis de los hallazgos empíricos en el aula y de las propuestas didácticas realizadas en los últimos años, facilitando el acceso de los profesores de estos instrumentos a estos datos. A partir de estos objetivos, se formularon las siguientes preguntas:

a) ¿Qué tendencias en investigación en el campo de la enseñanza de los ICFFV se hallan en las universidades españolas en los últimos 15 años?

b) ¿Qué universidades tienen una mayor producción de tesis sobre este tema?

c) ¿Cuáles son los hallazgos o aportaciones más relevantes de estas tesis?

\section{Método}

Se realizó un "estudio descriptivo mediante el análisis de documentos" (Osca-Lluch et al., 2013, p. 101). Para la selección de estos documentos, se hizo una "Búsqueda Avanzada" de tópicos en la base de datos Teseo entre el 3 y el 11 de junio de 2018. Se llevó a cabo un proceso que constó de cuatro fases:

1. Estimación previa de resultados. Se comenzó por realizar una estimación de posibles trabajos relacionados con los instrumentos de la familia del violín. Sin aplicar ningún filtro, se utilizaron los tópicos expuestos en el punto 2.1 para buscar en el campo "Título/Resumen" de la base de datos. En esta primera estimación, se constató que el número de tesis devueltas era excesivo para poder ser operativo.

\footnotetext{
${ }^{2}$ En este trabajo, se entiende por instrumentos de cuerda frotada a los pertenecientes a la familia del violín: violín, viola y violonchelo. A diferencia de los tres instrumentos mencionados, no se incluye al contrabajo, pues tradicionalmente se ha considerado como un instrumento de la familia de la viola da gamba. Aunque no se incluye en este trabajo y puesto que se trata de un instrumento de arco ampliamente utilizado, merece la pena especificarse que no se hallan tesis relacionadas con los procesos de enseñanza-aprendizaje del contrabajo en el aula en la base de datos Teseo.
}

@ROBERTO MACIÁN GONZÁLEZ. THE CONTENT OF THIS ARTICLE IS THE SOLE RESPONSIBILITY OF THE AUTHORS. THE REVISTA ELECTRÓNICA DE LEEME AND UNIVERSITAT DE VALĖNCIA ARE NOT LIABLE FOR ANY LEGAL ACTIONS THAT MAY ARISE INVOLVING THE ARTICLE'S CONTENT. REVISTA ELECTRÓNICA DE LEEME -LISTA ELECTRÓNICA EUROPEA DE MÚSICA EN LA EDUCACIÓN-HTTP://OJS.UV.ES/INDEX/PHP/LEEME/INDEX. ISSN: 1575-956. EDITORES: UNIVERSIDAD DE VALENCIA Y JESÚS TEJADA GIMÉNEZ. VISIBILIDAD DE ESTA REVISTA: SCOPUS, EMERGING SOURCES CITATION INDEX (CLARIVATE), EBSCO, CINDOC (CSIC), CITEFACTOR, COPAC, DIALNET, DICE (CSIC), DOAJ, E-REVISTAS (CSIC), EBSCO
PREMIER, ERIH+, GALE CENGAGE LEARNING, IN-RECS, IRESIE, LATINDEX, MIAR, OCLC WORLDCAT, RESH, REDIB, RILM CORE JOURNALS, SUDOC, ULRICHS, ESTA REVISTA ESTÁ PREMIER, ERIH+, GALE CENGAGE LEARNING, IN-RECS, IRESIE, LATINDEX, MIAR, OCLC WORLDCAT, RESH, REDIB, RILM CORE JOURNALS, SUDOC, ULRICHS, ESTA REVISTA
PUBLICADA CON EL APOYO INSTITUCIONAL DE REDIRIS-CONSEJO SUPERIOR DE INVESTIGACIONES CIENTIFICAS Y ES DE ACCESO LIBRE. CREATIVE COMMONS LICENSE 4.O BY 
2. Preselección de referencias (muestra 1). Se realizó una búsqueda con los criterios de inclusión/exclusión disponibles en la base de datos. También, se buscó en el campo "Título/Resumen" con los tópicos mencionados, pero se aplicaron los filtros de "Curso Académico" y "Palabra Clave" disponibles en la base de datos para reducir la muestra a estudiar.

3. Refinamiento de las referencias (muestra 2). Tras la búsqueda más detallada de la segunda fase, se analizó el contenido de los resúmenes de las tesis devueltas (Kantorski, 2015), incluyéndolas o excluyéndolas siguiendo los otros criterios de inclusión/exclusión. Los casos que ofrecían dudas a partir de la lectura del resumen también se incluyeron en la selección final de tesis a estudiar en profundidad.

4. Estudio de los trabajos seleccionados (muestra final). Se realizó a través del análisis del contenido vertido en las tesis seleccionadas.

Las fases 2 y 3 se repitieron en diciembre de 2018 para comprobar si se habían añadido nuevos trabajos en la base de datos. Al constatar que no se habían añadido nuevas tesis, no fue necesario volver a realizar la cuarta fase.

\subsection{Tópicos utilizados}

La búsqueda se realizó de distinta forma según la fase a la que se refiere:

1. Para realizar la estimación previa, se utilizaron los tópicos: "violín" (con y sin acento), "viola", "violonchelo", "violoncello", "instrumento de cuerda" e "instrumentos de cuerda".

2. Para la preselección de referencias (muestra 1), se utilizó el comodín *, posibilitando la devolución de todos los resultados referidos a los instrumentos musicales objeto de estudio. También, se siguió con los tópicos "instrumento de cuerda" e "instrumentos de cuerda".

\subsection{Criterios de inclusión/exclusión}

Se establecieron unos criterios para la inclusión o exclusión de trabajos en la muestra final. A excepción del año de defensa, todos los criterios vinieron definidos por los propósitos de la investigación:

- Disciplina. Se excluyeron todos los trabajos no relacionados con la música, la musicología o la enseñanza musical. Para ello, se introdujeron las palabras clave

@ROBERTO MACIÁN GONZÁLEZ. THE CONTENT OF THIS ARTICLE IS THE SOLE RESPONSIBILITY OF THE AUTHORS. THE REVISTA ELECTRÓNICA DE LEEME AND UNIVERSITAT DE VALĖNCIA ARE NOT LIABLE FOR ANY LEGAL ACTIONS THAT MAY ARISE INVOLVING THE ARTICLE'S CONTENT. REVISTA ELECTRÓNICA DE LEEME -LISTA ELECTRÓNICA EUROPEA DE MÚSICA EN LA EDUCACIÓN-HTTPP://OJS.UV.ES/INDEX/PHP/LEEME/INDEX. ISSN: 1575-956. EDITORES: UNIVERSIDAD DE VALENCIA Y JESÚS TEJADA GIMÉNEZ. VISIBILIDAD DE ESTA REVISTA: SCOPUS, EMERGING SOURCES CITATION INDEX (CLARIVATE), EBSCO, CINDOC (CSIC), CITEFACTOR, COPAC, DIALNET, DICE (CSIC), DOAJ, E-REVISTAS (CSIC), EBSCO
PREMIER, ERIH+, GALE CENGAGE LEARNING, IN-RECS, IRESIE, LATINDEX, MIAR, OCLC WORLDCAT, RESH, REDIB, RILM CORE JOURNALS, SUDOC, ULRICHS, ESTA REVISTA ESTAA PUBLICADA CON EL APOYO INSTITUCIONAL DE REDIRIS-CONSEJO SUPERIOR DE INVESTIGACIONES CIENTIFICAS Y ES DE ACCESO LIBRE. CREATIVE COMMONS LICENSE 4.0 BY 
"[620306] - Música y Musicología" en el buscador de la base de datos a través del buscador de palabras clave de Tesauro, disponible en el propio portal de Teseo.

- Temática. Se excluyeron todas las tesis no relacionadas con los procesos de enseñanzaaprendizaje en la práctica de aula. Así, se excluyeron los estudios de corte historiográfico, organológico o compositivo. Asimismo, se excluyeron los trabajos con un enfoque artístico, médico, sociológico o histórico. También, se excluyeron los trabajos referidos a otros instrumentos de otras familias de instrumentos de cuerda, los centrados exclusivamente en los resultados de interpretación y los trabajos versados en la práctica en casa.

- Fecha de defensa. Se incluyeron las tesis defendidas desde el año 2003 hasta el 2018 (ambos inclusive). Cuando se abordan las revisiones de literatura, se considera necesario que las fuentes sean actualizadas. Puesto que este trabajo pretende ofrecer una herramienta útil para investigadores y profesores, se amplió el intervalo de 5 años que algunos autores recomiendan para que las referencias estén actualizadas (Martín y Lafuente, 2017). Por esta razón, se decidió triplicar esta fecha y revisar las tesis defendidas los últimos 15 años, ofreciendo un abanico más amplio de la investigación en la enseñanza de los ICFFV. En el campo "Curso académico" de la base de datos, se introdujo el intervalo entre 2002-03 y 2018-19, evitando posibles sesgos de información por el rango de fechas.

- Población estudiada. Se excluyeron los trabajos relacionados con los músicos profesionales o con los profesores y propia práctica.

\section{Resultados}

Tras realizar la primera estimación sin ningún tipo de filtro, la base de datos devolvió alrededor de 4.000 tesis. La muestra 1 estuvo integrada por 67 trabajos. Posteriormente, la muestra 2 estuvo formada por un total de 23 trabajos. Finalmente, realizado el estudio de esta segunda muestra, quedó una muestra final de 8 tesis realmente relacionadas con el objeto de estudio de este trabajo. Debido a la naturaleza y propósitos de estas 8 tesis, se pueden dividir en dos categorías: 1) investigaciones realizadas en (y sobre) la práctica en el aula; y 2) propuestas didácticas (o didáctico-metodológicas) innovadoras para la enseñanza de los ICFFV (puestas en práctica o no).

$\mathrm{Al}$ realizar las búsquedas, se encontraron varios problemas. Un primer obstáculo fue que la base de datos no devolvió las referencias de algunas tesis que eran conocidas por el investigador. La razón por la que esto sucedió no se conoce, pero la base de datos devolvió un trabajo más en la búsqueda de diciembre: una tesis defendida en enero de 2016. Además de esto, uno de los registros devueltos por Teseo mostraba un título acortado frente al expresado en la portada de la tesis. Otro problema que apareció fue que Teseo devolvió varios trabajos para más de uno de los tópicos utilizados. El último escollo fue que hubo varios casos en los que el título

@ROBERTO MACIÁN GONZÁLEZ. THE CONTENT OF THIS ARTICLE IS THE SOLE RESPONSIBILITY OF THE AUTHORS. THE REVISTA ELECTRÓNICA DE LEEME AND UNIVERSITAT DE VALĖNCIA ARE NOT LIABLE FOR ANY LEGAL ACTIONS THAT MAY ARISE INVOLVING THE ARTICLE'S CONTENT. REVISTA ELECTRÓNICA DE LEEME -LISTA ELECTRÓNICA EUROPEA DE MÚSICA EN LA EDUCACIÓN-HTTP://OJS.UV.ES/INDEX/PHP/LEEME/INDEX. ISSN: 1575-956. EDITORES: UNIVERSIDAD DE VALENCIA Y JESÚS TEJADA GIMÉNEZ. VISIBILIDAD DE ESTA REVISTA: SCOPUS, EMERGING SOURCES CITATION INDEX (CLARIVATE), EBSCO, CINDOC (CSIC), CITEFACTOR, COPAC, DIALNET, DICE (CSIC), DOAJ, E-REVISTAS (CSIC), EBSCO
PREMIER, ERIH+, GALE CENGAGE LEARNING, IN-RECS, IRESIE, LATINDEX, MIAR, OCLC WORLDCAT, RESH, REDIB, RILM CORE JOURNALS, SUDOC, ULRICHS, ESTA REVISTA ESTÁ PUBLICADA CON EL APOYO INSTITUCIONAL DE REDIRIS-CONSEJO SUPERIOR DE INVESTIGACIONES CIENTIFICAS Y ES DE ACCESO LIBRE. CREATIVE COMMONS LICENSE 4.0 BY 
de la tesis era muy vago o demasiado "literario" y/o el resumen casi inexistente o muy inexacto. Esto propició que en la muestra 2 se incluyesen tesis descartables a priori, pero se incluyeron tratando de evitar posibles sesgos por falta de información.

\subsection{Instrumentos, niveles educativos y agrupación en clase}

Como se ha comentado, la muestra final estuvo integrada por 8 tesis, seis dedicadas al violín y dos al violonchelo (Figura 1). Conviene destacar que ningún estudio de la muestra final estuvo destinado al aprendizaje de la viola o la enseñanza de los ICFFV de forma general. De los trabajos dedicados al violín, hubo 3 investigaciones realizadas en el aula y 3 propuestas didácticas. Los trabajos relacionados con el violonchelo fueron dos propuestas didácticas.

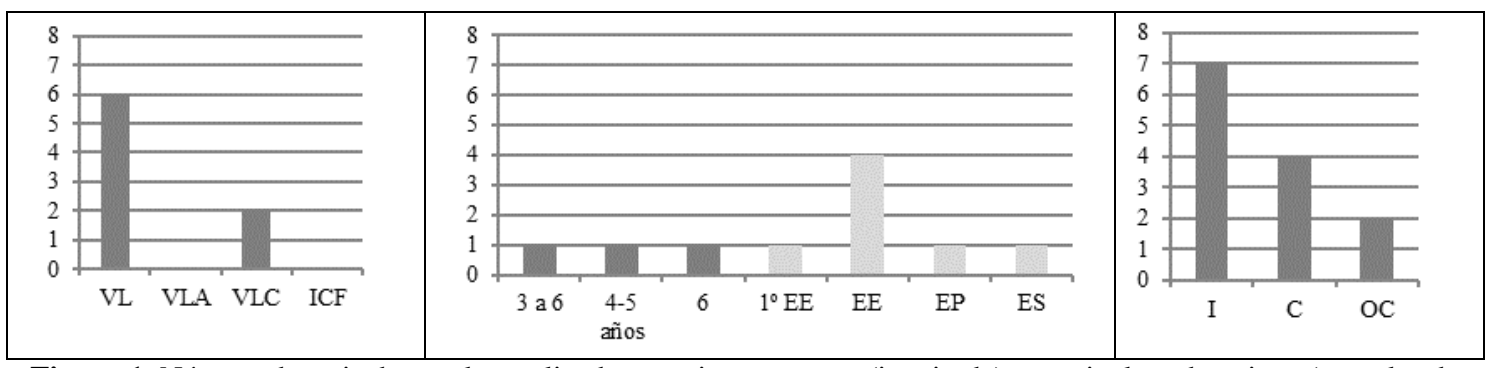

Figura 1. Número de tesis doctorales realizadas: por instrumentos (izquierda), por niveles educativos (escuelas de música en gris oscuro y conservatorios en gris claro) (centro) y por tipo de agrupación en clase (derecha). VL: violín; VLA: viola; VLC: violonchelo; ICFFV: Instrumentos de cuerda frotada de la familia del violín; EE: enseñanza elemental; EP: enseñanza profesional; ES: enseñanza superior; I: individual; C: colectiva; OC: otro tipo de formación colectiva

Por lo general, los estudios analizados se realizaron en los conservatorios (Figura 1). De estos, tres tesis se dedicaron exclusivamente a las enseñanzas elementales, siendo una de ellas para el primer curso y dos para los cuatro cursos que comprenden dichas enseñanzas. Dos tesis más se centraron en este tipo de centros, una realizada para alumnado de enseñanzas elementales y profesionales y otro para enseñanzas superiores. En escuelas de música, se llevaron a cabo dos tesis: una para alumnado de 4-5 años y otra para alumnado de 6 años. Sólo hubo una tesis de aplicación en los dos tipos de centro: en escuela de música desde los 3 años y en conservatorio hasta los 12 años. Conviene destacar que una de las propuestas didácticas, destinada a alumnado de enseñanzas elementales y profesionales, se diseñó para alumnado con discapacidad visual.

En cuanto al tipo de agrupamiento en clase, predominan los estudios realizados en clases individuales. Sólo una de las tesis tuvo como objeto exclusivo la clase colectiva y hubo dos trabajos realizados para clases individuales y colectivas, uno realizado para el violín y el otro para el violonchelo. Además, dos mostraron de forma indirecta su aplicabilidad en otras asignaturas del currículo como son clases de música de cámara (enseñanza colectiva).

@ROBERTO MACIÁN GONZÁLEZ. THE CONTENT OF THIS ARTICLE IS THE SOLE RESPONSIBILITY OF THE AUTHORS. THE REVISTA ELECTRÓNICA DE LEEME AND UNIVERSITAT DE VALĖNCIA ARE NOT LIABLE FOR ANY LEGAL ACTIONS THAT MAY ARISE INVOLVING THE ARTICLE'S CONTENT. REVISTA ELECTRÓNICA DE LEEME -LISTA ELECTRÓNICA EUROPEA DE MÚSICA EN LA EDUCACIÓN-HTTP://OJS.UV.ES/INDEX/PHP/LEEME/INDEX. ISSN: 1575-956. EDITORES: UNIVERSIDAD DE VALENCIA Y JESÚS TEJADA GIMÉNEZ. VISIBILIDAD DE ESTA REVISTA: SCOPUS, EMERGING SOURCES CITATION INDEX (CLARIVATE), EBSCO, CINDOC (CSIC), CITEFACTOR, COPAC, DIALNET, DICE (CSIC), DOAJ, E-REVISTAS (CSIC), EBSCO
PREMIER, ERIH+, GALE CENGAGE LEARNING, IN-RECS, IRESIE, LATINDEX, MIAR, OCLC WORLDCAT, RESH, REDIB, RILM CORE JOURNALS, SUDOC, ULRICHS, ESTA REVISTA ESTÁ PREMIER, ERIH+, GALE CENGAGE LEARNING, IN-RECS, IRESIE, LATINDEX, MIAR, OCLC WORLDCAT, RESH, REDIB, RILM CORE JOURNALS, SUDOC, ULRICHS, ESTA REVISTA
PUBLICADA CON EL APOYO INSTITUCIONAL DE REDIRIS-CONSEJO SUPERIOR DE INVESTIGACIONES CIENTIFICAS Y ES DE ACCESO LIBRE. CREATIVE COMMONS LICENSE 4.O BY 


\subsection{Tamaño de muestra y enfoque utilizado}

Los tamaños de las muestras y los enfoques encontrados fueron muy variados, encontrándose pocas coincidencias entre las distintas tesis. Conviene destacar que, dependiendo del tipo de estudio, las muestras fueron utilizadas para llevar a cabo la investigación en sí misma o para validar las propuestas didácticas. En los casos de investigaciones basadas en la práctica, las muestras fueron pequeñas, desde 9 hasta 13 estudiantes. Para validar las propuestas, las muestras fueron muy dispares, desde 3 casos concretos de estudiantes hasta la recogida de datos de 40. La validación de las propuestas que lo necesitaron fue realizada por un número variable de expertos. En este punto, se debe remarcar que las tesis fueron muy distintas, sólo encontrándose una cierta semejanza en las escasas muestras de los trabajos de investigación en el aula. En cuanto al tipo de enfoque, el más utilizado fue el cualitativo, siendo la InvestigaciónAcción utilizada en tres de las investigaciones. El resto utilizaron diseños distintos en cada caso, con enfoque cualitativo o cuantitativo y variables en su duración.

\subsection{Universidades y fecha de lectura}

A diferencia de otras disciplinas o áreas, no se encontraron universidades claramente identificadas con la enseñanza de los ICFFV (Figura 2). El único caso en el que se han llevado a cabo dos de estos estudios ha sido la Universidad Rey Juan Carlos (URJC). El resto de trabajos son únicos en su universidad: Universidad Autónoma de Madrid (UAM), Universidad Católica San Antonio de Murcia (UCAM), Universidad de Las Palmas de Gran Canaria (ULPGC), Universidad de Murcia (UM), Universidad Rovira i Virgili (URV) y Universitat de València (Estudi General) (UV).

La primera tesis relacionada con los procesos de enseñanza-aprendizaje de los ICFFV se realizó en $2012^{3}$ (Figura 2). A partir de ese año, se produjo un incremento de este tipo de estudios, llegando a defenderse 5 de ellos en el año 2017. Estos datos indican que se trata de un objeto de estudio novedoso en las universidades españolas, pero que cuenta con un interés creciente en los últimos años. Sin embargo, no se ha defendido ninguna tesis de este tipo en el año 2018.

\footnotetext{
${ }^{3}$ Nagore (2005) explica que la fecha provista en Teseo puede no coincidir con la fecha de lectura, sino con la fecha de entrega. No obstante, para este trabajo, se ha utilizado la "Fecha de lectura" contenida en la base de datos asumiendo que es correcta.
}

@ROBERTO MACIÁN GONZÁLEZ. THE CONTENT OF THIS ARTICLE IS THE SOLE RESPONSIBILITY OF THE AUTHORS. THE REVISTA ELECTRÓNICA DE LEEME AND UNIVERSITAT DE VALĖNCIA ARE NOT LIABLE FOR ANY LEGAL ACTIONS THAT MAY ARISE INVOLVING THE ARTICLE'S CONTENT. REVISTA ELECTRÓNICA DE LEEME -LISTA ELECTRÓNICA EUROPEA DE MÚSICA EN LA EDUCACIÓN-HTTP://OJS.UV.ES/INDEX/PHP/LEEME/INDEX. ISSN: 1575-956. EDITORES: UNIVERSIDAD DE VALENCIA Y JESÚS TEJADA GIMÉNEZ. VISIBILIDAD DE ESTA REVISTA: SCOPUS, EMERGING SOURCES CITATION INDEX (CLARIVATE), EBSCO, CINDOC (CSIC), CITEFACTOR, COPAC, DIALNET, DICE (CSIC), DOAJ, E-REVISTAS (CSIC), EBSCO
PREMIER, ERIH+, GALE CENGAGE LEARNING, IN-RECS, IRESIE, LATINDEX, MIAR, OCLC WORLDCAT, RESH, REDIB, RILM CORE JOURNALS, SUDOC, ULRICHS, ESTA REVISTA ESTA PREMIER, ERIH+, GALE CENGAGE LEARNING, IN-RECS, IRESIE, LATINDEX, MIAR, OCLC WORLDCAT, RESH, REDIB, RILM CORE JOURNALS, SUDOC, ULRICHS, ESTA REVISTA
PUBLICADA CON EL APOYO INSTITUCIONAL DE REDIRIS-CONSEJO SUPERIOR DE INVESTIGACIONES CIENTIFICAS Y ES DE ACCESO LIBRE. CREATIVE COMMONS LICENSE 4 . O BY 


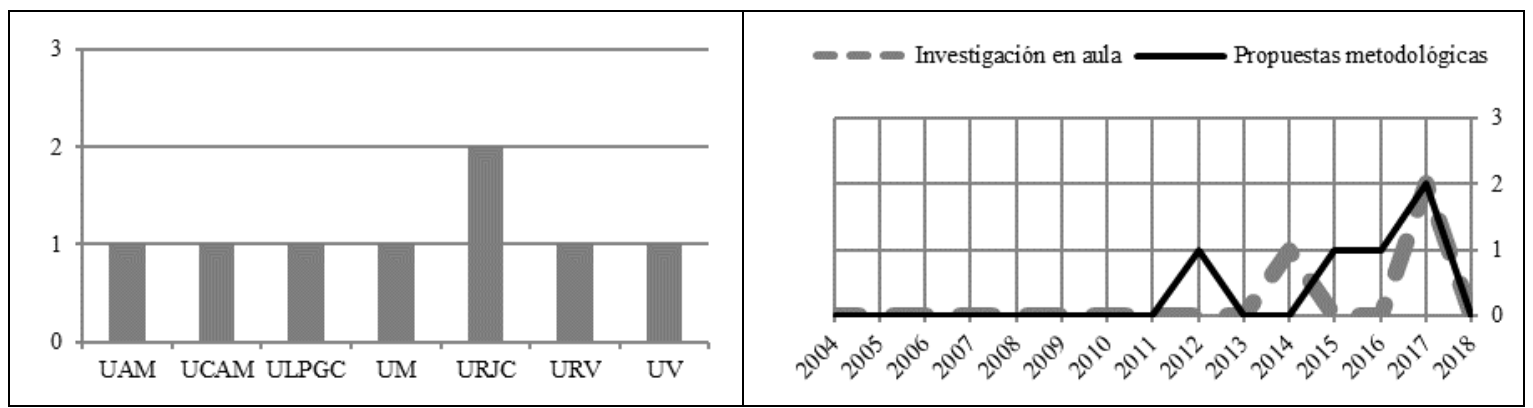

Figura 2. Producción de las universidades (izquierda) y fecha de defensa (derecha)

\subsection{Hallazgos y utilidades para los profesores de los ICFFV}

A continuación, se muestran las propuestas didácticas de algunos investigadores para la enseñanza de los ICFFV (Tabla 1). Estos trabajos han sido validados aplicándolos en el aula o a través de cuestionarios y/o entrevistas. Los hallazgos más destacables en las investigaciones empíricas en aula sobre el proceso de enseñanza-aprendizaje de los ICFFV se muestran en la Tabla 2. Conviene remarcar que en algunos de los trabajos excluidos también se hallan informaciones útiles para la enseñanza de los ICFFV, pero se han obtenido a través de otro tipo de investigaciones.

Tabla 1. Nuevas propuestas didácticas para la enseñanza de los ICFFV

Instrumento Alumnado ${ }^{4}$

Violín

Iniciación (desde 3 años) y/o EE.EE.
Descripción de las propuestas y temas del marco teórico sobre los que se fundamentan

Descripción: Monroy (2016) propone una colección de tres materiales didácticos. Para ello, analiza una serie de materiales de iniciación al violín de 24 autores desde 1950 hasta 2013 y realiza una propuesta propia, pero utilizando las TIC como apoyo y guía para el proceso de enseñanza-aprendizaje. Los dos primeros volúmenes se centran en las cuestiones más básicas de la técnica: postura, cuerdas al aire, golpes de arco y primera posición. También aporta una colección de piezas pequeñas. En el tercer volumen, se adentra en las escalas y los cambios de posición, así como ejercicios técnicos específicos y algunas obras. Aunque utiliza las TIC, ofrece una versión imprimible de los tres materiales. Temas: 1) métodos musicales del siglo XX: definición de método, métodos de educación musical relevantes y principales métodos de enseñanza del violín; 2) educación musical en España: breve introducción a la legislación más relevante y tipologías de centros de educación musical; y 3 ) sociedad del conocimiento: TIC, sistemas de gestión del aprendizaje (e-learning), dispositivos móviles (m-learning) y uso de las aplicaciones (apps) en educación.

\footnotetext{
${ }^{4}$ De forma general, las Enseñanzas Elementales se cursan entre los 8 y los 12 años, las Profesionales entre los 12 y los 18 años y las Enseñanzas Superiores a partir de los 18 años.
}

@ROBERTO MACIÁN GONZÁLEZ. THE CONTENT OF THIS ARTICLE IS THE SOLE RESPONSIBILITY OF THE AUTHORS. THE REVISTA ELECTRÓNICA DE LEEME AND UNIVERSITAT DE VALĖNCIA ARE NOT LIABLE FOR ANY LEGAL ACTIONS THAT MAY ARISE INVOLVING THE ARTICLE'S CONTENT. REVISTA ELECTRÓNICA DE LEEME -LISTA ELECTRÓNICA EUROPEA DE MÚSICA EN LA EDUCACIÓN-HTTP://OJS.UV.ES/INDEX/PHP/LEEME/INDEX. ISSN: 1575-956. EDITORES: UNIVERSIDAD DE VALENCIA Y JESÚS TEJADA GIMÉNEZ. VISIBILIDAD DE ESTA REVISTA: SCOPUS, EMERGING SOURCES CITATION INDEX (CLARIVATE), EBSCO, CINDOC (CSIC), CITEFACTOR, COPAC, DIALNET, DICE (CSIC), DOAJ, E-REVISTAS (CSIC), EBSCO PREMIER, ERIH+, GALE CENGAGE LEARNING, IN-RECS, IRESIE, LATINDEX, MIAR, OCLC WORLDCAT, RESH, REDIB, RILM CORE JOURNALS, SUDOC, ULRICHS, ESTA REVISTA ESTÁ PUBLICADA CON EL APOYO INSTITUCIONAL DE REDIRIS-CONSEJO SUPERIOR DE INVESTIGACIONES CIENTIFICAS Y ES DE ACCESO LIBRE. CREATIVE COMMONS LICENSE 4.O BY 

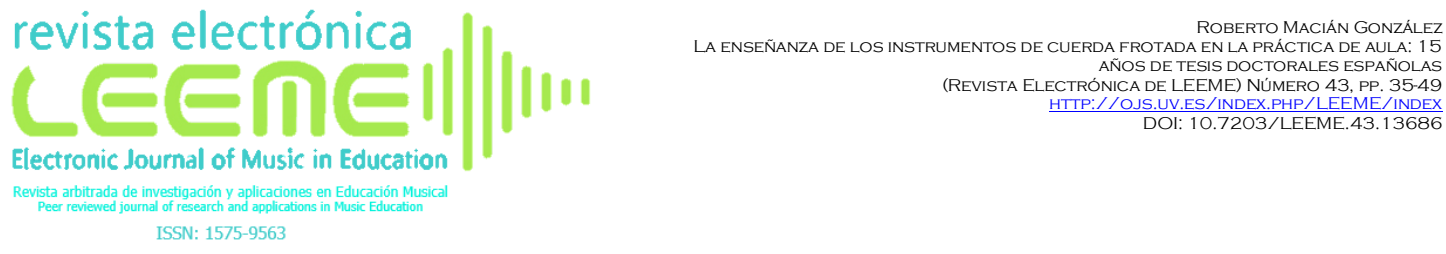

Tabla 1. Nuevas propuestas didácticas para la enseñanza de los ICFFV

Instrumento

Alumnado ${ }^{4}$

Violonchelo

Enseñanzas

elementales (EE.EE.)
Descripción de las propuestas y temas del marco teórico sobre los que se fundamentan

Descripción: García Sánchez (2015) propone una programación para el aula de violonchelo en los conservatorios elementales de Murcia. A través del análisis de los 6 materiales didácticos más utilizados en los conservatorios de esta comunidad autónoma, plantea una secuencia didáctica (objetivos, contenidos, metodología, evaluación, materiales didácticos), desarrollando cada curso por separado y también la clase colectiva. Divide los contenidos por trimestres, propone la enseñanza de la técnica basándose en profesores relevantes, plantea los métodos/libros de estudios y el repertorio secuenciado por cursos. Ofrece dos materiales imprimibles: uno para el trabajo de arco y una recopilación de canciones populares para dos violonchelos. Temas: 1) el violonchelo: características del instrumento y del arco; 2) enseñanza del violonchelo: las escuelas violonchelísticas, el instrumento en España, descripción y evolución de la legislación española y métodos de enseñanza del instrumento; 3) el violonchelo en Murcia: profesorado y alumnado relevante a lo largo de la historia; y 4) estilos de enseñanza y aprendizaje en educación musical.

Descripción: Campà (2012) sugiere una batería de cuestiones prácticas a la hora de realizar las clases colectivas de violonchelo. Entre éstas, aborda los recursos materiales y una amplia gama de actividades a realizar, incluyendo actividades creativas y actividades técnicas, todas desglosadas según las distintas posibilidades de las que se puede disponer en un conservatorio. Aporta una colección de fichas imprimibles en las que especifica los objetivos, contenidos, criterios metodológicos, evaluación y recursos necesarios. Al final de la tesis concreta los contenidos para cada curso. Temas: 1) cuestiones generales de aprendizaje participativo en clases colectivas; 2) distribución espacial (también aborda otras cuestiones como repertorio, clima de trabajo, creatividad o responsabilidad en el mismo apartado); 3) evaluación; y 4) actividades de conjunto: ritmo y audición, lectoescritura, improvisación, conceptos, grabación, técnica, golpes de arco, mano izquierda, estimular la creatividad, capacidad de concentración, ergonomía, interpretación, y repentización.

Violín

Enseñanzas elementales y profesionales (Discapacidad visual)

Descripción: Sánchez (2017) propone todo un "compendio de buenas prácticas, recursos y estrategias metodológicas para alumnos de violín con deficiencia visual". En este, aborda cuestiones organizativas, estrategias docentes, recursos materiales y humanos, planteamiento de las clases, afrontar las audiciones y las clases colectivas o el trabajo con los pianistas acompañantes. Esta serie de cuestiones las concreta tanto para las enseñanzas elementales como profesionales, así como realiza sugerencias para el resto de asignaturas en el conservatorio. Temas: 1) discapacidad visual: concepto, grados y tipos; 2) el sistema Braille: generalidades, antecedentes, lectura y escritura; 3) la Organización Nacional de Ciegos Españoles (ONCE): historia y funcionamiento; 4) la enseñanza escolar con alumnos con discapacidad visual: cuestiones generales, enseñanza de la lecto-escritura, las distintas áreas y enseñanza en la adolescencia; y 5) práctica y educación de los músicos ciegos: musicografía Braille, otras musicografías, técnicas y recursos materiales para alumnado con discapacidad visual.

Violín

Enseñanzas

Descripción: Solovieva (2017) propone un nuevo sistema para la mejora de los problemas relacionados con la producción de sonido en el violín, basado en la comparación de la técnica de la producción sonora del violín con las posibilidades técnicas del canto. A partir de las observaciones de los alumnos, algunos de los problemas a los que propone soluciones mediante ejercicios relacionados con el canto son: falta de la continuidad de tensión durante el fraseo o el paso de arco, exceso de rigidez en el brazo o en los dedos del arco, problemas en la realización del vibrato o con los intervalos de gran distancia entre notas. Ante problemas en la técnica, sugiere utilizar siempre una aproximación interdisciplinar para tratar de solucionarlos. Temas: realiza un estado de la cuestión sobre la aplicación del canto en la enseñanza del violín (materiales musicológicos, métodos de violín y biografías, junto con artículos y tesis doctorales).

Fuente: Elaboración propia

@ROBERTO MACIÁN GONZÁLEZ. THE CONTENT OF THIS ARTICLE IS THE SOLE RESPONSIBILITY OF THE AUTHORS. THE REVISTA ELECTRÓNICA DE LEEME AND UNIVERSITAT DE VALĖNCIA ARE NOT LIABLE FOR ANY LEGAL ACTIONS THAT MAY ARISE INVOLVING THE ARTICLE'S CONTENT. REVISTA ELECTRÓNICA DE LEEME -LISTA ELECTRÓNICA EUROPEA DE MÚSICA EN LA EDUCACIÓN-HTTP://OJS.UV.ES/INDEX/PHP/LEEME/INDEX. ISSN: 1575-956. EDITORES: UNIVERSIDAD DE VALENCIA Y JESÚS TEJADA GIMÉNEZ. VISIBILIDAD DE ESTA REVISTA: SCOPUS, EMERGING SOURCES CITATION INDEX (CLARIVATE), EBSCO, CINDOC (CSIC), CITEFACTOR, COPAC, DIALNET, DICE (CSIC), DOAJ, E-REVISTAS (CSIC), EBSCO PREMIER, ERIH+, GALE CENGAGE LEARNING, IN-RECS, IRESIE, LATINDEX, MIAR, OCLC WORLDCAT, RESH, REDIB, RILM CORE JOURNALS, SUDOC, ULRICHS, ESTA REVISTA ESTA
PUBLICADA CON EL APOYO INSTITUCIONAL DE REDIRIS-CONSEJO SUPERIOR DE INVESTIGACIONES CIENTIFICAS YES DEACCESO LIBRE, CREATIVE COMMONS LICENSE 4.O BY 
Tabla 2. Hallazgos empíricos relevantes sobre la enseñanza-aprendizaje de los ICFFV

Instrumento
Alumnado

\section{Violín}

4-5 años

\section{Planteamiento teórico inicial de las investigaciones y hallazgos más relevantes}

Planteamiento: El aprendizaje inicial del violín puede realizarse utilizando tres aproximaciones: 1) aprox. de Mano Derecha (MD), basada en la práctica del agarre, producción de sonido y distintos patrones de arco, retrasando el aprendizaje de los sonidos producidos con la mano izquierda; 2) aprox. de Mano Izquierda (MI), que practica los distintos sonidos del violín, la digitación y la entonación siempre en pizzicato, postergando el manejo de arco; y 3) aprox. de Ambas manos (AM), que combina el aprendizaje de las dos manos de forma alterna o simultánea.

Hallazgos: En ambas franjas de edad, se obtuvieron elevados rendimientos y muy buenos resultados en la práctica con el instrumento. A los cuatro años, se recomienda utilizar una aproximación didáctica que utilice las dos manos desde el principio si los alumnos cuentan con un fuerte acompañamiento familiar en clase. Sin este acompañamiento, se sugiere utilizar una aproximación de mano derecha, pues resultó más motivadora. A los cinco años, se recomienda utilizar la aproximación de ambas manos (Macián, 2017).

Violín Planteamiento: La plasticidad neuronal podría explicarse como la capacidad del cerebro que 6 años le permite adaptarse al contexto mediante la creación o poda de conexiones neuronales. Esta capacidad, a su vez, modifica el aprendizaje. Esta plasticidad está más desarrollada en los primeros años de vida $\mathrm{y}$, entre los 6 y los 7 años, se produce un importante avance madurativo derivado de esta plasticidad. Esto repercute en la motricidad fina y la lectoescritura, permitiendo iniciarse instrumentalmente antes de lo establecido en las enseñanzas musicales oficiales.

Hallazgos: Se puede comenzar a tocar el violín a los 6 años, 2 años antes de lo establecido en el sistema escolar musical. Esto aprovecha la mayor plasticidad neuronal que tienen los niños a esta edad. El hecho de realizar la acción docente en centros no reglados no implica una falta de consecución de objetivos supuestos para centros reglados. Adaptarse a las necesidades individuales mediante atención periódica y tener en cuenta la motivación de los alumnos pueden compensar las deficiencias propias de la edad (García Díaz, 2017).

Violín Planteamiento: Los métodos tradicionales pueden entenderse como aquellos que provienen $1^{\circ}$ EE.EE. (+/-8 años) de las distintas tradiciones pedagógicas heredadas de los siglos anteriores y que se basan en el desarrollo de la técnica del violín, obviando otras cuestiones relacionadas con otras áreas de lo que supone el aprendizaje del instrumento. Este tipo de métodos siguen utilizándose en muchos conservatorios, siendo escasos los centros en los que se emplean métodos para desarrollar la creatividad junto con la técnica instrumental al mismo tiempo.

Hallazgos: Se puede desarrollar la creatividad simultáneamente a la adquisición de las destrezas técnicas instrumentales. De hecho, los alumnos centrados en el método propuesto para el trabajo conjunto de técnica y creatividad obtuvieron un mejor desarrollo técnico que aquellos que se habían iniciado con uno de los métodos considerados tradicionales (Gallego, 2014).

Fuente: Elaboración propia

\section{Discusión}

Este trabajo supone una pequeña aportación a la investigación de áreas específicas en territorios concretos (Morales et al., 2017), abordando temáticas muy delimitadas, tal y como ya se venía haciendo en otros países (Kantorski, 1995; Preston y Humphreys, 2007). Según los resultados obtenidos, los procesos de enseñanza-aprendizaje de los ICFFV son una temática poco tratada en las tesis doctorales españolas. Esta carencia ya se había apuntado en estudios anteriores refiriéndose a todas las disciplinas instrumentales (Gillanders y Martínez, 2005). Por

@ROBERTO MACIÁN GONZÁLEZ. THE CONTENT OF THIS ARTICLE IS THE SOLE RESPONSIBILITY OF THE AUTHORS. THE REVISTA ELECTRÓNICA DE LEEME AND UNIVERSITAT DE VALĖNCIA ARE NOT LIABLE FOR ANY LEGAL ACTIONS THAT MAY ARISE INVOLVING THE ARTICLE'S CONTENT. REVISTA ELECTRÓNICA DE LEEME - LISTA ELECTRÓNICA EUROPEA DE MÚSICA EN LA EDUCACIÓN-HTTP://OJS.UV.ES/INDEX/PHP/LEEME/INDEX. ISSN: 1575-956. EDITORES: UNIVERSIDAD DE VALENCIA Y JESÚS TEJADA GIMÉNEZ. VISIBILIDAD DE ESTA REVISTA: SCOPUS, EMERGING SOURCES CITATION INDEX (CLARIVATE), EBSCO, CINDOC (CSIC), CITEFACTOR, COPAC, DIALNET, DICE (CSIC), DOAJ, E-REVISTAS (CSIC), EBSCO PREMIER, ERIH+, GALE CENGAGE LEARNING, IN-RECS, IRESIE, LATINDEX, MIAR, OCLC WORLDCAT, RESH, REDIB, RILM CORE JOURNALS, SUDOC, ULRICHS, ESTA REVISTA ESTÁ
PUBLICADA CON EL APOYO INSTITUCIONAL DE REDIRIS-CONSEJO SUPERIOR DE INVESTIGACIONES CIENTIFICAS Y ES DE ACCESO LIBRE. CREATIVE COMMONS LICENSE 4.O BY 
esta razón, puede considerarse como una línea de investigación novedosa en los estudios de doctorado sobre música, suponiendo un cambio en los enfoques de investigación en los últimos años (Nagore, 2005). De hecho, este tipo de investigaciones se concentran en los últimos 5 años.

En cuanto al instrumento objeto de estudio, el violín es el más estudiado, seguido por el violonchelo (Kantorski, 1995). A diferencia de los hallazgos de Kantorski, la viola o la práctica de los ICFFV de forma conjunta (enseñanza heterogénea en la que intervienen 2, 3 o más tipos de instrumentos) no se han abordado en las universidades españolas. Esto puede deberse a las distintas tradiciones que se hallan en cada país. En España, la enseñanza individual es la tradicional. Por el contrario, en Estados Unidos hay una gran tradición en enseñanza grupal heterogénea en los distintos niveles educativos. También conviene destacar la gran variedad de métodos de investigación y muestras encontradas, hecho que pone de manifiesto las múltiples posibilidades y enfoques metodológicos.

Resulta destacable que las tesis estudiadas no se han llevado a cabo en las universidades más productivas en investigación musical. A diferencia de otros trabajos (Nagore, 2005; Gillanders y Martínez, 2005), la universidad más prolífica ha sido la Universidad Rey Juan Carlos. Otras universidades que no aparecen como las más productivas en el campo musical, pero sí surgen de esta investigación son: Universidad Católica San Antonio de Murcia, Universidad de Las Palmas de Gran Canaria, Universidad de Murcia y Universidad Rovira i Virgili. Las dos únicas universidades que siguen la tendencia en cuanto a producción es la Universidad Autónoma de Madrid y la Universitat de València.

Finalmente, merece atención el trabajo realizado con la propia base de datos Teseo. Por un lado, debe tenerse en cuenta la necesidad de realizar las búsquedas con unos criterios exactos y bien definidos. De lo contrario, la búsqueda de trabajos se convierte en una tarea imposible. Por otro, deben abordarse los problemas encontrados a la hora de trabajar con esta base de datos. Un primer problema fue que no se devolvió uno de los trabajos (muestra 1) tras varios meses después de su defensa (Fernández, Torralbo, Vallejo, 2008). Esto pone de manifiesto la lenta actualización de los datos contenidos en Teseo (Nagore, 2005) y la necesidad de triangulación de resultados mediante el acceso a varias fuentes. Otro problema es el relacionado con los títulos introducidos en Teseo, que pueden contener errores (Oriol, 2009, 2012). Esto se constató con una de las tesis, cuyo título fue acortado para la base de datos frente al expuesto en el documento de la tesis. Esta problemática pudo deberse a que el título fuese demasiado largo y que no cupiese al introducirlo en el registro de Teseo. Finalmente, es necesario estandarizar los resúmenes de las tesis doctorales. Este hecho facilitaría en gran medida las tareas de revisión y la búsqueda concreta de trabajos.

@ROBERTO MACIÁN GONZÁLEZ. THE CONTENT OF THIS ARTICLE IS THE SOLE RESPONSIBILITY OF THE AUTHORS. THE REVISTA ELECTRÓNICA DE LEEME AND UNIVERSITAT DE VALĖNCIA ARE NOT LIABLE FOR ANY LEGAL ACTIONS THAT MAY ARISE INVOLVING THE ARTICLE'S CONTENT. REVISTA ELECTRÓNICA DE LEEME -LISTA ELECTRÓNICA EUROPEA DE MÚSICA EN LA EDUCACIÓN-HTTP://OJS.UV.ES/INDEX/PHP/LEEME/INDEX. ISSN: 1575-956. EDITORES: UNIVERSIDAD DE VALENCIA Y JESÚS TEJADA GIMÉNEZ. VISIBILIDAD DE ESTA REVISTA: SCOPUS, EMERGING SOURCES CITATION INDEX (CLARIVATE), EBSCO, CINDOC (CSIC), CITEFACTOR, COPAC, DIALNET, DICE (CSIC), DOAJ, E-REVISTAS (CSIC), EBSCO
PREMIER, ERIH+, GALE CENGAGE LEARNING, IN-RECS, IRESIE, LATINDEX, MIAR, OCLC WORLDCAT, RESH, REDIB, RILM CORE JOURNALS, SUDOC, ULRICHS, ESTA REVISTA ESTÁ PUBLICADA CON EL APOYO INSTITUCIONAL DE REDIRIS-CONSEJO SUPERIOR DE INVESTIGACIONES CIENTIFICAS Y ES DE ACCESO LIBRE. CREATIVE COMMONS LICENSE 4.O BY 


\section{Conclusiones}

La investigación sobre los procesos de enseñanza-aprendizaje en el aula de instrumento debería ser una práctica habitual en conservatorios y escuelas de música, facilitando que los profesores se replanteasen sus enseñanzas. Sin embargo, se ha constatado que el sistema universitario español se encuentra en un estadio muy embrionario respecto a estas investigaciones. Según los resultados obtenidos en este artículo, queda evidenciada la carencia que se encuentra sobre este tipo de estudios en España. Por ello, es necesario aumentar su número y objetos de indagación, permitiendo avanzar hacia una didáctica más reflexiva y fundamentada en investigación. Con esto, se abandonaría una práctica instalada en creencias y tradición que, en ocasiones, se muestra ineficaz y frustrante tanto para profesorado como para alumnado.

\section{Referencias}

Campà, C. M. (2012). Propuesta metodológica para la enseñanza de la clase de conjunto en el grado elemental aplicada al violoncello (Tesis Doctoral no publicada). Universidad Rey Juan Carlos, Madrid.

Escorcia, T. A. (2008). El análisis bibliométrico como herramienta para el seguimiento de publicaciones científicas, tesis y trabajos de grado (Trabajo Fin de Grado no publicado). Pontificia Universidad Javeriana, Bogotá.

Fernández, A., Torralbo, M. y Vallejo, M. (2008). Revisión y prospectiva de la producción española en tesis doctorales de pedagogía (1976-2006). Revista de Investigación Educativa, 26 (1), 191-207.

Gallego, J. L. (2014). El desarrollo de la creatividad mediante la improvisación en el violín: Análisis comparativo entre dos metodologías de enseñanza (Tesis Doctoral no publicada). Universidad Rey Juan Carlos, Madrid.

García Díaz, E. F. (2017). El violín: un instrumento para el desarrollo musical y cognitivo desde la infancia (Tesis Doctoral no publicada). Universidad Autónoma de Madrid, Madrid.

García Sánchez, E. M. (2015). Del violonchelo en la región de Murcia: Una aproximación histórica y una propuesta didáctica para la enseñanza de grado elemental (Tesis Doctoral no publicada). Universidad de Murcia, Murcia.

Gillanders, C. y Martínez, P. (2005). La investigación en el ámbito musical. Música y Educación, 64, 85-104.

@ROBERTO MACIÁN GONZÁLEZ. THE CONTENT OF THIS ARTICLE IS THE SOLE RESPONSIBILITY OF THE AUTHORS. THE REVISTA ELECTRÓNICA DE LEEME AND UNIVERSITAT DE VALĖNCIA ARE NOT LIABLE FOR ANY LEGAL ACTIONS THAT MAY ARISE INVOLVING THE ARTICLE'S CONTENT. REVISTA ELECTRÓNICA DE LEEME -LISTA ELECTRÓNICA EUROPEA DE MÚSICA EN LA EDUCACIÓN-HTTP://OJS.UV.ES/INDEX/PHP/LEEME/INDEX. ISSN: 1575-956. EDITORES: UNIVERSIDAD DE VALENCIA Y JESÚS TEJADA GIMÉNEZ. VISIBILIDAD DE ESTA REVISTA: SCOPUS, EMERGING SOURCES CITATION INDEX (CLARIVATE), EBSCO, CINDOC (CSIC), CITEFACTOR, COPAC, DIALNET, DICE (CSIC), DOAJ, E-REVISTAS (CSIC), EBSCO
PREMIER, ERIH+, GALE CENGAGE LEARNING, IN-RECS, IRESIE, LATINDEX, MIAR, OCLC WORLDCAT, RESH, REDIB, RILM CORE JOURNALS, SUDOC, ULRICHS, ESTA REVISTA ESTÁ PREMIER, ERIH+, GALE CENGAGE LEARNING, IN-RECS, IRESIE, LATINDEX, MIAR, OCLC WORLDCAT, RESH, REDIB, RILM CORE JOURNALS, SUDOC, ULRICHS, ESTA REVISTA
PUBLICADA CON EL APOYO INSTITUCIONAL DE REDIRISCONSEJO SUPERIOR DE INVESTIGACIONES CIENTIFICAS Y ES DE ACCESO LIBRE. CREATIVE COMMONS LICENSE 4.0 BY 
Kantorski, V. J. (1995). A content analysis of doctoral research in string education, 1936-1992. Journal of Research in Music Education, 43 (4), 288-297. doi: 10.2307/3345728

Macián, R. (2017). Contraste de dos aproximaciones didácticas en la iniciación al violín: una investigación en el aula con niños de 4-5 años (Tesis Doctoral no publicada). Universitat de València, Valencia.

Martín, S. G. y Lafuente. V. (2017). Referencias bibliográficas: indicadores para su evaluación en trabajos científicos. Investigación Bibliotecológica, 31 (71), 151-180. doi:10.22201/iibi.0187358xp.2017.71.57814

Martínez, L. J. (2013). Cómo buscar y usar información científica. Guía para estudiantes universitarios 2013. Santander: Universidad de Cantabria. Recuperado de https://goo.gl/xXOFsy

Ministerio de Educación, Cultura y Deporte. (2017). Teseo. Gestión de tesis doctorales. Manual de usuario doctorando. Actualización versión 4.1.9. Recuperado de https://www.educacion.gob.es/teseo/teseo/abrirAyuda.do

Monroy, R. (2016). Estudio y análisis comparativo de la metodología contemporánea del violín. Propuesta metodológica para la didáctica del violín en las enseñanzas de iniciación y/o elemental (Tesis Doctoral no publicada). Universidad de las Palmas de Gran Canaria, Las Palmas de Gran Canaria.

Morales, Á., Ortega, E., Conesa, E. y Ruiz-Esteban, C. (2017). Análisis bibliométrico de la producción científica en Educación Musical en España. Revista Española de Pedagogía, 75 (268), 399-414. doi: 10.22550/REP75-3-2017-07

Nagore, M. (2005). Tesis doctorales. La investigación musical en España: Situación actual y perspectivas de futuro. Revista de Musicología, 28 (2), 1451-1470. doi: 10.2307/20798139

Narejos, A. (2018). Tesis de música en España [Base de datos]. Recuperado de http://www.tesisdemusica.es/database.html

Oriol, N. (2009). La investigación musical en España: Tesis doctorales y temática en la última década. Eufonía, 45, 59-87.

Oriol, N. M. (2012). Temática de tesis doctorales de Música en España (1978-2011). Música y Educación, 92 (4), 58-94.

Osca-Lluch, J., Haba, J., Fonseca, S., Civera, C. y Tortosa, F. (2013). Tesis doctorales españolas sobre análisis bibliométrico en Psicología. Aula Abierta, 41(2), 99-110. Recuperado de https://bit.ly/2QkHBQU

@ROBERTO MACIÁN GONZÁLEZ. THE CONTENT OF THIS ARTICLE IS THE SOLE RESPONSIBILITY OF THE AUTHORS. THE REVISTA ELECTRÓNICA DE LEEME AND UNIVERSITAT DE VALĖNCIA ARE NOT LIABLE FOR ANY LEGAL ACTIONS THAT MAY ARISE INVOLVING THE ARTICLE'S CONTENT. REVISTA ELECTRÓNICA DE LEEME -LISTA ELECTRÓNICA EUROPEA DE MÚSICA EN LA EDUCACIÓN-HTTPP://OJS.UV.ES/INDEX/PHP/LEEME/INDEX. ISSN: 1575-956. EDITORES: UNIVERSIDAD DE VALENCIA Y JESÚS TEJADA GIMÉNEZ. VISIBILIDAD DE ESTA REVISTA: SCOPUS, EMERGING SOURCES CITATION INDEX (CLARIVATE), EBSCO, CINDOC (CSIC), CITEFACTOR, COPAC, DIALNET, DICE (CSIC), DOAJ, E-REVISTAS (CSIC), EBSCO
PREMIER, ERIH+, GALE CENGAGE LEARNING, IN-RECS, IRESIE, LATINDEX, MIAR, OCLC WORLDCAT, RESH, REDIB, RILM CORE JOURNALS, SUDOC, ULRICHS, ESTA REVISTA ESTÁ PREMIER, ERIH+, GALE CENGAGE LEARNING, IN-RECS, IRESIE, LATINDEX, MIAR, OCLC WORLDCAT, RESH, REDIB, RILM CORE JOURNALS, SUDOC, ULRICHS, ESTA REVISTA
PUBLICADA CON EL APOYO INSTITUCIONAL DE REDIRIS-CONSEJO SUPERIOR DE INVESTIGACIONES CIENTIFICAS Y ES DE ACCESO LIBRE. CREATIVE COMMONS LICENSE 4 . B BY 
Pastor, P. (2002). La investigación educativa musical. Eufonía, 26, 84-88.

Preston, K. Y. y Humphreys, J. T. (2007). Historical research on music education and music therapy: Doctoral dissertations of the Twentieth Century. Journal of Historical Research in Music Education, 29 (1), 55-73. doi:10.1177/153660060702900106

Sánchez, L. (2017). La didáctica del violín en alumnos con discapacidad visual: Compendio de buenas prácticas, recursos y estrategias metodológicas para una educación inclusiva en las enseñanzas elementales y profesionales de música (Tesis Doctoral no publicada). Universidad Católica San Antonio, Murcia.

Solovieva, O. (2017). Teaching violin to higher level students: the analogy and benefits of vocal approach (Tesis Doctoral no publicada). Universitat Rovira i Virgili, Tarragona. 